\title{
Clipless laparoscopic cholecystectomy by Harmonic shears
}

\author{
Hany Mohamed El- Barbary, MD FRCSI ; Mohamed Aly Nada, MD; \\ Ahmed El-Sayed Mourad, MD; Hanna Habib Hanna, MD; \\ Sameh Maaty, MD; Hussein Abdul-Alim Boushnak, MD \\ Department of General Surgery, Ain Shams University, Cairo, Egypt.
}

(Presented in the 11th Congress of the Modern Egyptian Surgical Society, MESS, October 2008, Sukhna, Egypt)

\begin{abstract}
Background: The primary use of the Harmonic scalpel in laparoscopic cholecystectomy has been for the division of the cystic artery and liver bed dissection. In this study, we evaluate the safety \& efficacy of Harmonic scalpel as the sole instrument for division of the cystic duct and artery as well as dissection of the liver bed (clipless cholecystectomy).

Methods: A prospective, non randomized clinical trial was conducted on 21 consecutive patients with symptomatic gall bladder stones who underwent laparoscopic cholecystectomy (LC) in the surgical department, Ain Shams University Hospital, over a period of six months. In all patients, Harmonic scalpel was the sole instrument used to seal and divide the cystic artery and duct as well as for dissection of the gall bladder bed. The operative time, operative blood losses, intraoperative and postoperative complications especially bile leaks, hospital stay as well as cost were evaluated. A short term postoperative follow up was done for all patients for one month.

Results: Twenty (95.2\%) successful clipless laparoscopic cholecystectomies were conducted with no open conversion. One patient (4.8\%) early in the series developed postoperative bile leakage, which was managed by ERCP. There were no other complications in our series. The operative time ranged from 60-100 minutes (median=80min.). The hospital stay was 1-3 days (median= 2 days). No gall bladder perforations occurred and the quality of the operative field was excellent with traces or minimal blood loss.

Conclusion: Clipless laparoscopic cholecystectomy by Harmonic scalpel (shears) is safe and effective to carry out after completing the required learning curve.
\end{abstract}

\section{Introduction:}

Gallstone disease remains the most common disease of the digestive system and laparoscopic cholecystectomy (LC) is the gold standard treatment of gallstones because of its many known advantages in comparison with 'open' abdominal surgery. ${ }^{1,2}$ It is one of the most common surgical procedures performed. ${ }^{3}$

The primary use of the Harmonic scalpel in laparoscopic cholecystectomy has been for the division of the cystic artery and liver bed dissection. Advancements in the Harmonic scalpel blade tip now provide a safe alternative for reliable ultrasonic division and closure of the cystic duct. The ultrasonically activated (Harmonic Scalpel, HS) allows performing dissection and coagulation with a minimal thermal side effect for surrounding tissues, unlike the electro coagulation. This innovative method of cutting tissue was based upon the coagulating and cavitational effects provided by a rapidly vibrating blade contacting various tissues. 4

The principle is transforming of the electric power into mechanical longitudinal movement of the working part of the instrument, by piezoelectric transducer situated in the hand piece. ${ }^{5}$ The resulting decrease in temperatures, smoke, and lateral tissue damage placed the Harmonic scalpel in contrast to the effects seen with the more traditional electro surgery/cautery. In addition, the elimination of inadvertent, sometimes unrecognized, electrical arcing injuries with their potentially hazardous sequelae supported the role of the Harmonic scalpel as a potentially safer 
instrument for tissue dissection. ${ }^{4}$

In this study, we evaluate the safety \& efficacy of Harmonic scalpel (HS) as the sole instrument for division of the cystic duct and artery as well as dissection of the liver bed i.e. Clipless cholecystectomy (CLC).

\section{Patients and methods:}

A prospective, non randomized clinical trial was conducted on 21 consecutive patients with symptomatic gall bladder stones who underwent laparoscopic cholecystectomy (LC) in the surgical department of Ain Shams University Hospital, over a period of six months. In all patients, Harmonic scalpel ("Ethicon Endo-Surgery", USA) was the sole instrument used to seal and divide the cystic artery and duct as well as for dissection of the gall bladder bed. The patients were 16 females and 5 males $(M / F=1: 3.2)$, their ages ranged between $21-61$ years (median=41yrs). All had symptomatic gall bladder stones proved by abdominal ultrasound. No intraoperative cholangiograms were done as any patient presenting with symptoms of choledocholithiasis or biliary pancreatitis was excluded from the study and was sent for evaluation by preoperative endoscopic retrograde cholangio pancreatography (ERCP) and treated accordingly. The operative time, operative blood loss, intraoperative complications as bleeding, gall bladder perforation, bile spillage \pm stone spillage, conversion rate, postoperative complications especially bile leaks or fistulae, bleeding or unrecognized injuries were recorded. The hospital stay was calculated as well as costs.

All patients had postoperative follow up for four weeks after discharge, in the form of clinical examination, repeating liver functions and abdominal ultrasound as outpatient.

\section{Surgical technique:}

Patients received a single dose of prophylactic intravenous antibiotic on induction of general anesthesia. Patients were positioned in the usual anti- Trendelenburg position with right side tilt and open Hasson's technique was employed for pneumo-peritoneum. A four port technique was employed in all patients and a disposable $5 \mathrm{~mm}$ Harmonic ACE -"Ethicon
Endo-Surgery", USA- was used as the sole instrument for dissection and coagulation; i.e. no use of Maryland, Titanium clips or clip applier and no use of electrocautery. Dissection of fibrinous adhesions as well as structures in Callot's triangle was carried out in the usual manner using the Harmonic scalpel setting the ultrasonic generator on maximum power and dissection was carried on, keeping with the principles of gentle traction and counter traction to display the anatomical structures Figure(1).

Before cystic duct division, the power of the ultrasonic generator was set to the minimum power. The cystic duct was skeletonized Figure(2) and the cystic duct/ common bile duct confluence identified. Before division of the cystic duct Figure $(\mathbf{3 , 4})$ the traction applied to Hartmann's pouch was decreased to release the tension on the cystic duct, then the Harmonic scalpel blades were firmly applied for 20-30 seconds till complete division of the duct, allowing for a safe coagulum to form and seal the stump. The distal stump was also sealed in this way, preventing spillage of bile. The cystic artery was next identified, skeletonized and divided in the same manner with no tension to avoid bleeding. The gall bladder was next removed in a systematic way keeping the active blade of the Harmonic into view and close to the gall bladder wall Figure(5,6). Drains were routinely used in all procedures Figure(7). Postoperatively patients were allowed soft diet after the first motion and then discharged home when well enough, usually after 24 hours. Our results are presented and discussed below. 


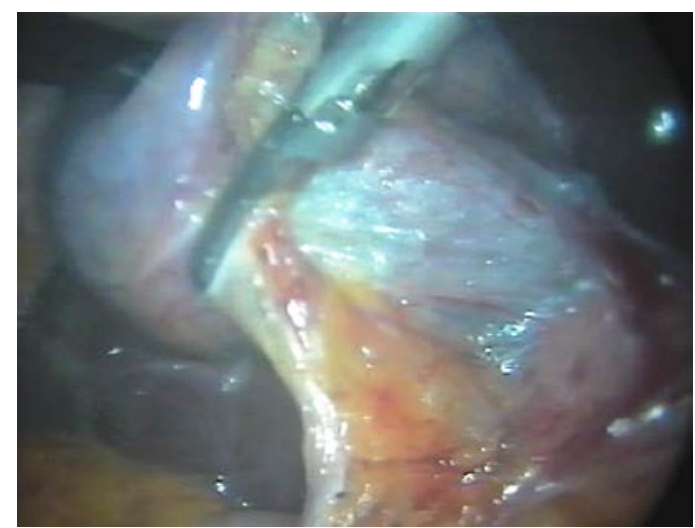

Figure (1): Dissection of adhesions.

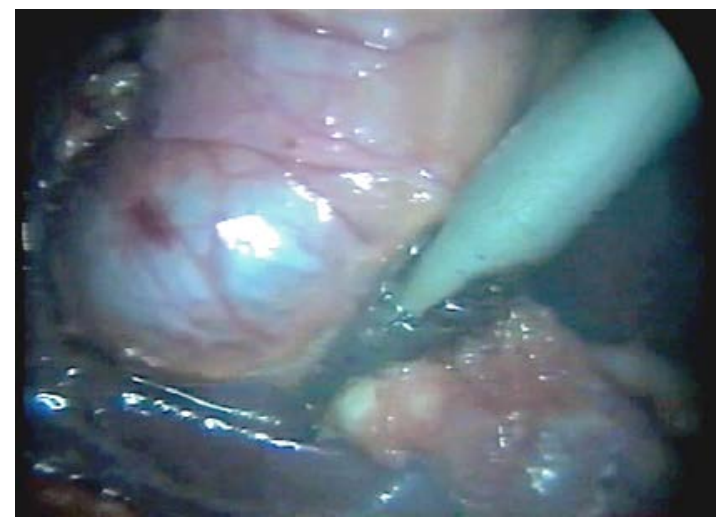

Figure (3): Division of cystic duct.

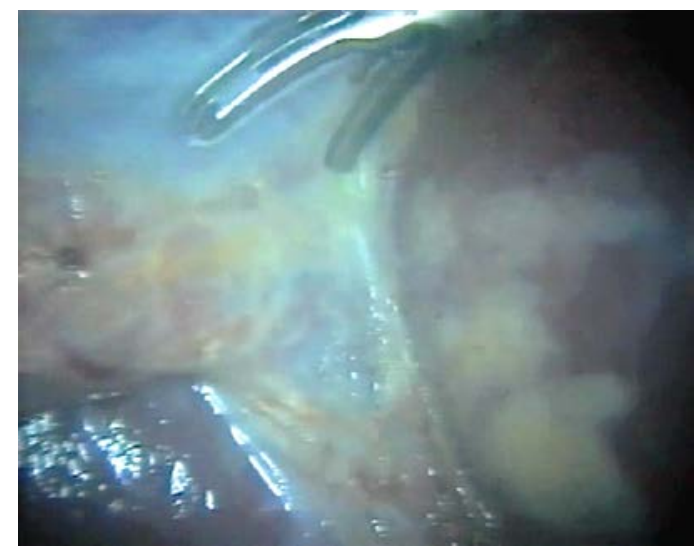

Figure (5): Dissection of GB bed.

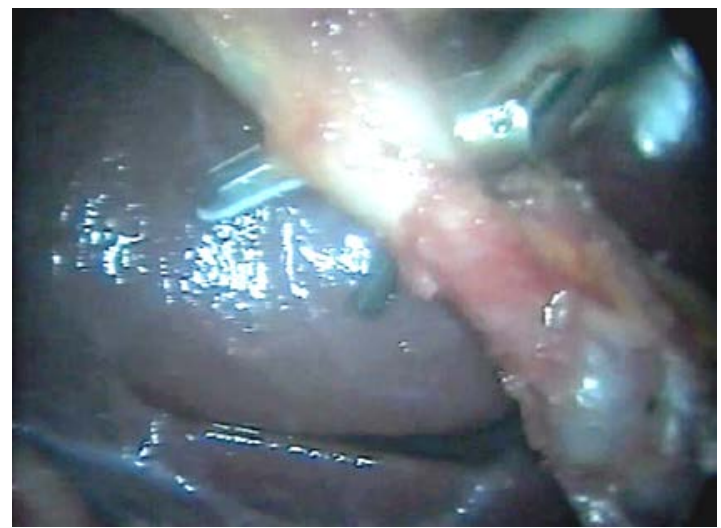

Figure (2): Skeletonization of cystic duct.

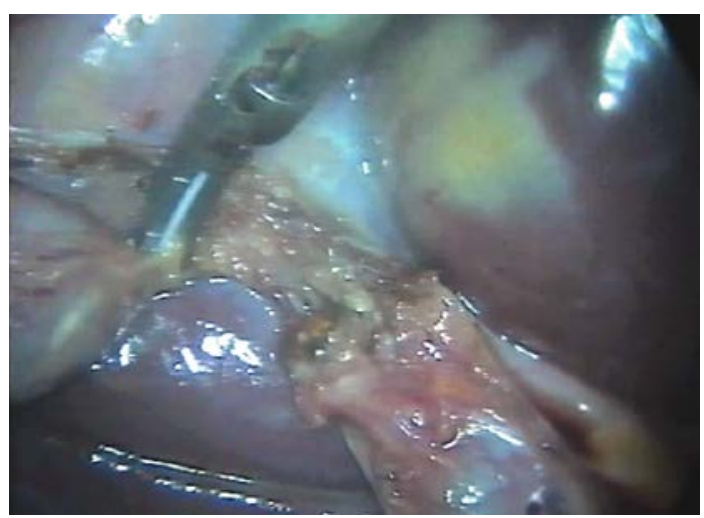

Figure (4): Sealing of the duct \& artery.

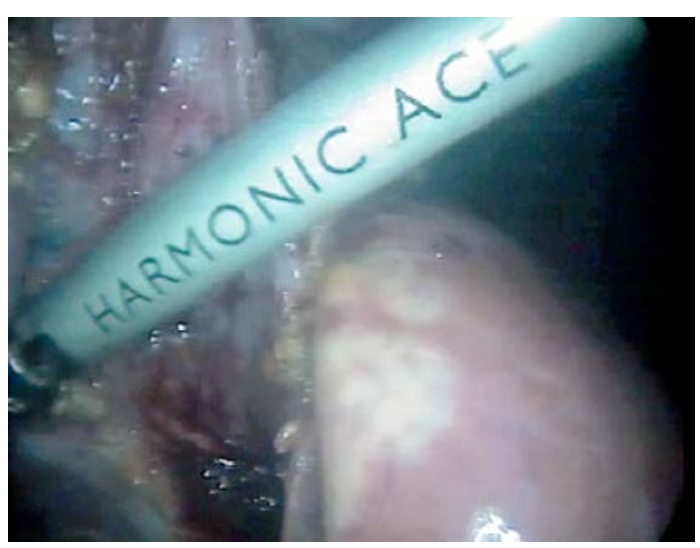

Figure (6): Complete removal of GB by $H S$.

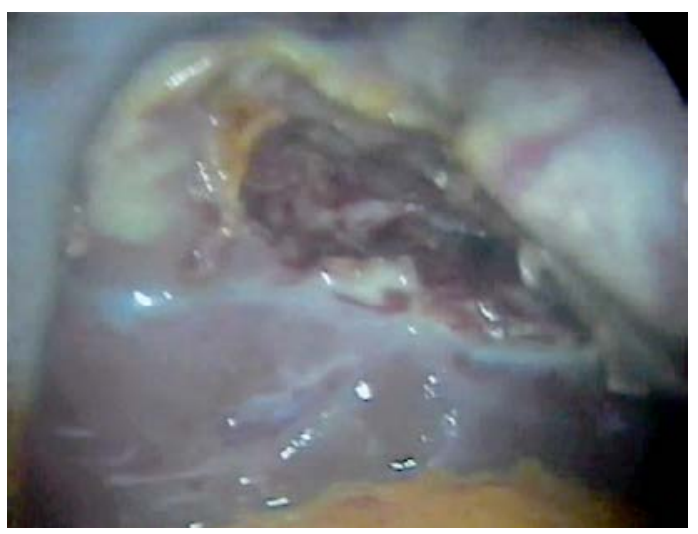

Figure (7): GB bed after cholecystectomy by HS. 


\section{Results:}

The patients were 16 females and 5 males, their ages ranged between 21- 61 years (median=41yrs). Twenty $(95.2 \%)$ successful clipless laparoscopic cholecystectomies (CLC) were completed with no open conversion Table(1). One patient (4.8\%) early in the series developed postoperative bile leakage on the second postoperative day and it was confirmed by ultrasound and managed by ERCP. The operative time ranged from 60-100 minutes (median $=80 \mathrm{~min}$ ). No gall bladder perforations or bile spillage occurred in any patient $(0 \%)$ and the quality of the operative field was excellent with traces or minimal blood loss. There was no postoperative bleeding or other complications in our series. The hospital stay was 1-3 days (median $=2$ days). When a drain was placed, the amount of the fluid collected after $24 \mathrm{hrs}$ ranged from $35-75 \mathrm{ml}$ serosanguinous exudate and the drain was subsequently removed. The exception was in the third patient in this series who developed bile leak on the second postoperative day (400 $\mathrm{ml}$ of bile in the drain). This was successfully managed by immediate ERCP \& stenting and the patient was discharged well next day and the suction drain subsequently removed 3 days later in the outpatient clinic. There were no operative or postoperative mortalities in our series $(0 \%)$.

The cost of the $5 \mathrm{~mm}$ Harmonic ACE "Ethicon Endo-Surgery", USA- is EGP 3500 and the cost of LC alone is about EGP1700. Each Harmonic ACE pair of blades was used up to six times.

The postoperative follow up of 19 patients was completed (90.5\%). Two of our patients $(9.5 \%)$ did not come for follow up after 2 weeks. None of the nineteen patients in our series revealed any sign of a missed bile duct injury, bowel injury or intra-abdominal collection on ultrasound and all had normal liver functions.

Table (1): Patient characteristics and data.

\begin{tabular}{|c|c|}
\hline Patient Characteristics & \\
\hline No & 21 \\
\hline Age & Median $=41$ \\
\hline $\mathrm{M}: \mathrm{F}$ & $1: 3.2$ \\
\hline \multicolumn{2}{|l|}{ Intraoperative } \\
\hline OR time & Median $=80 \mathrm{Min}$ \\
\hline Blood loss & Trace-minimal \\
\hline OR field & Excellent \\
\hline GB perforation & Non \\
\hline \multicolumn{2}{|l|}{ Postoperative } \\
\hline Bleeding & Non \\
\hline \multirow[t]{2}{*}{ Drain } & $35-75 \mathrm{Ml} / 24 \mathrm{Hr}$ \\
\hline & $400 \mathrm{Ml} / 24 \mathrm{Hr}$ \\
\hline Bile leaks $\quad \mathrm{N}=1$ & $1(4.8 \%)$ \\
\hline Conversion & Non \\
\hline Mortality & Non \\
\hline Follow up completed & 19 Patients out of $21(90.5 \%)$ \\
\hline
\end{tabular}




\section{Discussion:}

Advanced laparoscopic procedures have necessitated the development of new technology for vascular control. Suture ligation can be time-consuming and cumbersome during laparoscopic dissection. Titanium clips have been used for hemostasis, and recently plastic clips and energy sources such as ultrasonic coagulating shears and bipolar thermal energy devices have become popular. ${ }^{6}$

The Harmonic scalpel (HS) was introduced into clinical use nearly a decade ago. ${ }^{4}$ In Egypt, it was introduced few years ago and the latest laparoscopic version; Harmonic Ace ("Ethicon Endo-Surgery", USA) was introduced into practice at Ain Shams University Hospitals about one year back. The use of the HS brings a series of advantages in comparison to other electromagnetic forms of energy (electroscalpel, laser). HS cuts and coagulates with the same effectiveness of the electro-scalpel but, unlike this, it doesn't introduce risks of wandering currents. ${ }^{2}$

HS contributes to a cleaner and smoke-free field of operation and it reduces the operative time, the bleeding and the costs of the operation without an increase of the complications and of the percentages of 'open' conversion. ${ }^{2}$ This was apparent in all of our cases.

The Harmonic ACE produces the least thermal damage in the tissues. Energy related tissue damage is not only related to blade temperature, but is the result of several factors including blade temperature, transection time, tissue properties, and the vascularity of each transected tissue. ${ }^{7}$ In an early comparative analysis by Matthews et al in 2001, the mean thermal spread was $3.5 \mathrm{~mm}$ for ultrasonic coagulating shears and $13.4 \mathrm{~mm}$ for electro thermal bipolar vessel sealer $(\mathrm{P}=0.0002) .{ }^{8}$ Therefore, in our series there were no incidents of gall bladder perforations or thermal injuries of other important structures. The operative time was within the accepted range and became shorter with every case (60 min down from 80 min) due to getting more used to the instrument. However it was shown to be double that reported in the literature $(32-42 \mathrm{~min}){ }^{4,9}$

The bile ducts of Luschka, or subvesical ducts, are small ducts which originate from the right hepatic lobe, course along the gallbladder fossa, and usually drain in the extrahepatic bile ducts. Injuries to these ducts are the second most frequent cause of postcholecystectomy bile leaks. Bile leaks remain a significant cause of morbidity for patients undergoing this procedure. These occur in 0.2$2 \%$ of cases. ${ }^{3}$ In contrast, in our series, there was no evidence of bile ducts of Luschka on ERCP in the third patient who developed a biliary fistula on the second postoperative day (drain output $=400 \mathrm{ml}$ of bile), instead, and after reviewing the operative video, it was due to incomplete sealing of the cystic duct stump owing to excessive traction on the gall bladder and the resulting rapid cutting of the duct with insufficient coagulation. In an ex-vivo study, the mean cystic duct bursting pressure after ultrasonic coagulating shears was $278 \mathrm{~mm}$ Hg. ${ }^{8}$ This again emphasizes the importance of the learning curve and the importance of understanding the physical properties of the device as stated by Hüscher et al. ${ }^{1}$

The cost of the $5 \mathrm{~mm}$ Harmonic ACE "Ethicon Endo-Surgery", USA- is currently about EGP 3500 and can be used for up to six times in laparoscopic cholecystectomies. We used three successive instruments in this series i.e. EGP 580 per patient. On the other hand, the overall cost of a single laparoscopic cholecystectomy using Titanium clips (cost about EGP 90 per patient) were less. On the contrary, the use of Harmonic Ace enhances the procedure, as stated by Westervelt, ${ }^{4}$ so that indirect savings could be attributed to replacing three different instruments with one (the Harmonic scalpel replacing the Maryland dissector, the Titanium clips and their applier as well as the electro dissecting instrument being a spatula or hook dissector). All of the above resulted in less distraction of the surgical team by less instrument changing. However the increased total cost is yet an unresolved matter in our developing nation. Our results were close to the study by Bessa et al, who stated that Harmonic scalpel provides a superior alternative to the currently used high-frequency monopolar technology in terms of shorter operative time and lower incidence of gallbladder perforation. ${ }^{9}$ In conclusion, clipless laparoscopic cholecystectomy by Harmonic scalpel is safe and effective to carry out after completing the required learning curve. 


\section{References:}

1- Hüscher C, Lirici M, Di Paola M, et al: Laparoscopic cholecystectomy by ultrasonic dissection without cystic duct and artery ligature. Surg Endosc 2003; 17(3): 442-451.

2- Minutolo V, Gagliano G, Rinzivillo C, Li Destri G, et al: Usefullness of the ultrasonically activated scalpel in laparoscopic cholecystectomy: our experience and review of literature. G Chir 2008; 29(5): 242-245.

3- Spanos CP, Syrakos T: Bile leaks from the duct of Luschka (subvesical duct): A review. Langenbeck's Arch Surg 2006; 391(5): 441-447.

4- Westervelt J: Clipless cholecystectomy: Broadening the role of the harmonic scalpel. JSLS 2004; 8(3): 283-285.

5- Harrell G, Kercher K, Heniford B: Energy sources in laparoscopy. Semin Laparosc Surg 2004; 11: 201-209.
6- Harold L, Pollinger H, Matthews D, et al: Comparison of ultrasonic energy, bipolar thermal energy, and vascular clips for the hemostasis of small, medium, and largesized arteries. Surg Endosc 2003; 17(8): 1228-1230.

7- Phillips CK, Hruby GW, Durak E, et al: Tissue response to surgical energy devices. Urology 2008; 71(4): 744-748.

8- Matthews BD, Pratt BL, Backus CL, et al: Effectiveness of the ultrasonic coagulating shears, LigaSure vessel sealer, and surgical clip application in biliary surgery: a comparative analysis. Am Surg 2001; 67(9): 901-906.

9- Bessa S, Al-Fayoumi T, Katri K, Awad A: Clipless laparoscopic cholecystectomy by ultrasonic dissection. J Laparoendosc Adv Surg Tech A 2008; 18(4): 593-598. 